\title{
A Modern Appraisal of Ancient Etruscan Herbal Practices
}

\author{
${ }^{1}$ A. P. Harrison and ${ }^{2}$ E. M. Bartels \\ ${ }^{1}$ Biochemistry and Physiology, IBHV, The Royal Vet. and Agric. University \\ Grønnegaardsvej 7, 1870 Frederiksberg C, Copenhagen, Denmark \\ ${ }^{2}$ Copenhagen University Library, Noerre Allé 49, 2200 Copenhagen N, Denmark
}

\begin{abstract}
Individuals in antiquity would have been exposed to both cheese and red wine and perhaps as many as $10-40 \%$ of the population would have suffered at some time in their life from a migraine headache. Furthermore, individuals in antiquity would also have been exposed to their fair share of childhood stress and therefore would have been predisposed to arthritis in later life. However, it is perhaps more likely that rheumatoid arthritis occurred in ancient populations as a result of joint damage occurring from repetitive tasks such as milling grain, preparing hides etc. Finally, many of the symptoms treatable by the "Etruscan Herbal" would have been seen among those working with copper smelting and in view of the famous bronze work of the Etruscans, perhaps this herbal was directed more towards improving their health, rather than towards more ritual and magical practices. However, it is noteworthy that the "Etruscan Herbal" contains such plants as valerian and henbane, which with regard to their hypnotic and delirium-easing effects, respectively, may have been used in a more ritual and magical way by ancient herbalists and societies throughout the ancient Mediterranean. Without a doubt though, the application of the combined knowledge of the "Etruscan herbal" and its possible physiological effects, raises the issue of the importance of ancient treatments in today's society, particularly since we are still plagued by many of the same ailments as the ancient Etruscans.
\end{abstract}

Key words: Etruscan herbal, valerian, henbane, ancient treatments, physiological effects

\section{INTRODUCTION}

In a recent article entitled "An Etruscan Herbal?" (vol 5, Etruscan News, http://www.umass.edu/etruscannews/), Kyle P Johnson of New York University made reference to eleven plants understood in antiquity to pertain to Etruscan religious practice. These plants being valerian Valeriana officinalis (Valerianaceae), arum Arum italicum (Araceae), pimpernel, scarlet or blue Anagallis arvensis / caerulae (Primulaceae), gentian Gentiana acaulis (Gentianaceae), tuberous thistle Cnicus tuberosus (Asteraceae), thyme Thymus vulgaris (Lamiaceae), feverfew Tanacetum parthenium (Asteraceae), madder Rubia tinctoria (Rubiaceae), helichrysum Helichrysum stoechas (Asteraceae), henbane Hyoscyamus niger (Solanaceae) and rough bindweed Smilax aspera (Smilacaceae). The author concluded "further study of the use of these herbs may reveal more about Etruscan religious and herbal practices" and to this end it seems logical to identify not only the modern herbalists view of these eleven plants, but also the opinion of modern scientific knowledge.

This review aims to relate the biochemical composition of the "Etruscan herbal" to reported effects, taking into account current knowledge of physiology. When one refers to plants of medicinal importance, one often lists their active ingredients, which might be essential oils, alkaloids, bitters, flavonoids, tannins or glycosides. Essential oils are volatile and are often aromatic. They are most often effective on skin and mucous membranes and work well as expectorants. Alkaloids are a group of organic compounds that contain nitrogen. Many of these are poisonous, whilst others are very useful, for example codeine and morphine, which alleviate pain. Bitters are a mixed group of bitter-tasting compounds. In general they cause an increase in digestive secretions and improve digestion and appetite. Flavonoids, which are often yellow in colouration, are found in many plants. They affect, amongst other things, the wall of fine blood vessels and can be used to treat arteriosclerosis and high blood pressure. Tannins are found in many plants and as their name suggests, are the compounds used to tan leather. They can form a protective layer over infected skin and stop secretions or bleeding, whilst preventing bacteria infection of open wounds. Finally, glycosides are complex organic compounds that have a strong effect on heart function. Many can be very poisonous and perhaps the best known is digitalis

Corresponding Author: $\quad$ Dr. A.P. Harrison, Section of Biochemistry and Physiology, The Department of Animal and Veterinary Basic Sciences, The Royal Vet. \& Agric. University, Grønnegaardsvej 7, 1870 Frederiksberg C, Copenhagen, Denmark, Tel: +45 35282568 
from Foxglove, which is used to stimulate the heartbeat. Having given a general introduction to the field, we will now consider the "Etruscan herbal" one by one.

\section{Valerian}

Active ingredients: valepotriates, valeric acids, alkaloids, volatile oils and $\gamma$-Aminobutyric acid (GABA).

Uses: in homeopathic medicine to treat nervous tension, insomnia and nervous stomach and intestine problems, headaches, irritable bowel syndrome and eczema.

Negative side effects: None known.

This is one of the gentlest, non-toxic and nonaddictive tranquillisers known to mankind. It favourably influences the central nervous system (CNS) of the brain, acting as a natural relaxant to the higher nerve centres. It acts as a sedative and hypnotic and has been an effective calmative since Hippocratic times. It is as effective as benzodiazepines in relieving anxiety states and has no known side effects. Whilst attention has focused on the volatile oil and the valepotriates and their degradation products, the effects of the volatile oil cannot account totally for the action of the drug. Current thinking is that the overall effect of valerian is likely to involve the inhibitory transmitter Gammaaminobutyric acid (GABA) in the brain. Indeed, increased concentrations of GABA are associated with a decrease in CNS activity.

\section{Arum}

Active ingredients: saponins, alkaloids especially conisine.

Uses: anti-bacterial and anti-fungal, expectorant and anti-rheumatic.

Warning: poisonous if taken internally.

Negative side effects: None known.

In homoeopathy, a tincture of the root of Arum is effective against occipital headaches, which are often aggravated by damp weather. Such a tincture is also effective against dull pain in the brain arising from the least intellectual effort. Arum is also said to be effective against colic in the umbilical region associated with diarrhoea when applied locally to the skin. The saponin extract of Arum is an effective anti-bacterial agent with particular effect on S. epidermidis and S. aureus infections, whilst the alkaloid extract of Arum is an effective anti-microbial agent.

\section{Pimpernel}

Active ingredients: saponins, tannins, flavonoids, cucurbitacins.

Uses: in homeopathy to treat rashes and liver and gallbladder problems. It is also a diuretic, detoxifier, expectorant and induces sweating.

Warning: poisonous.

Negative side effects: Can alter serum glutamic oxaloacetic transaminase (SGOT) levels.
This plant was used to stimulate bile flow, to reduce nose-bleeds, act against snake bites, as well as improve cataracts and liver congestion. Although rarely used by herbalists today, it is sometimes used as a poultice in the treatment of joint pains. In folk medicine it has long been used to treat gallstones, cirrhosis of the liver, urinary infections, gout and rheumatism, a pattern of symptoms that suggest it may have a role in detoxifying the body. As an expectorant it was used to stimulate coughing which in turn brings up mucus and helps the lungs recover from colds and flu. Today there is considerable interest in the family of cucurbitacins due to their wide range of biological activity. A number of compounds, like cucurbitacins B, D, E and I from this group have recently been shown to be cytotoxic, hepatoprotective, anti-inflammatory and be beneficial for the cardiovascular system ${ }^{[1]}$.

\section{Gentian}

Active ingredients: glycosides gentiopicrin (bitters), amarogentin, gentianine.

Uses: in conventional and homeopathic medicine for digestive disorders, loss of appetite, flatulence, to stimulate digestive secretions and for liver and gallbladder problems.

Warning: A protected species.

Negative side effects: None known.

Gentian is known to stimulate liver and pancreatic function, to act as a haemopoietic, increasing red blood cell production and it has also been reported to act as an antidote to some types of food-poison (salmonella, shigella etc.). Root extracts of gentian have antifungal activity and are reported to stimulate phagocytic activity of human lymphocytes, indicating an immunostimulant activity. Choleretic properties have been documented for gentian and gentianine has been reported to possess anti-inflammatory activity. The bitters stimulate secretion of gastric juices and bile, thus aiding appetite and digestion. Finally, extracts of gentian are considered to be non-toxic and are generally well-tolerated.

\section{Tuberous Thistle}

Active ingredients: volatile alkaloids, cnicin.

Uses: as an emetic and emmenagogue.

Negative side effects: None known.

Whilst there are reports of no known medicinal uses of the tuberous thistle, the root is known to contain inulin, a starch that cannot be digested by humans and one that passes straight through the digestive system, often being fermented to produce flatulence. However, recent research has shown that inulin increases calcium absorption and calcium balance in man and that there is a link between inulin content of the diet and restoration of insulin sensitivity, reduction of risk of atherosclerosis, a reduced risk of osteoporosis and a reduced risk of colon cancer ${ }^{[2]}$. Finally, the compound 
cnicin has been shown in vitro to have a growth inhibitory effect on the cancerous cell lines MCF7 (breast) and OVCAR3 (ovarian) ${ }^{[3]}$.

\section{Thyme}

Active ingredients: essential oils, notably thymol, tannins, bitters, flavones, rosmarinic acid.

Uses: as expectorant and mild anaesthetic for coughs, whooping cough and acute and chronic bronchitis.

Negative side effects: With excessive use, dermal and membrane irritant and may create toxic symptoms like vomiting and cardiac arrest.

Thyme is effective against infections of the respiratory organs and urinary tract. It is also used against mouth ulcers (as a mouthwash), sore throats and against streptococcal infections. It's antitussive, that is to say capability to suppress coughing, expectorant and antispasmodic actions are considered to be associated with the volatile oils (thymol and carvacrol) and flavonoid constituents. In vitro antispasmodic activity of thyme has been associated with the phenolic components of the volatile oil and with the flavonoid constituents. Thymonin and circilineol, both flavonoids, have potent spasmoltyic activity in trachea preparations in vitro. Besides this, thymol, carvacrol and thyme oil have antifungal activity against a range of organisms and rosmarinic acid has an anti-inflammatory activity, also reducing the oedema associated with snake venom. However, thyme oil is a dermal and mucous membrane irritant. Toxic symptoms documented for thymol, include nausea, vomiting, gastric pain, headache, dizziness and cardiac and respiratory arrest. Thyme oil should therefore be used with considerable caution.

\section{Feverfew}

Active ingredients: sesquiterpene lactones, volatile oil, parthenolides.

Uses: mildly sedative, reducing inflammation as well as relieving migraine and other headaches, as well as arthritis and asthma.

Negative side effects: May affect contractions of blood vessels.

Used against inflammatory rheumatism and arthritis, but also for the prevention of migraine, Feverfew is also reported to be an antidote to mercurial poisoning. Feverfew extracts have been documented to inhibit platelet aggregation and prostaglandin, thromboxane and leukotriene production, although feverfew has also been reported to have no effect on cyclooxygenase - the mechanism by which nonsteroidal anti-inflammatory drugs inhibit prostaglandin production. Parthenolide markedly interferes with contractile and relaxant mechanisms in blood vessels, whilst antinociceptive properties have been reported for feverfew and parthenolide in vivo ${ }^{[4]}$.

\section{Madder}

This plant is of historic interest only, as a cholagogue, emmenagogue and diuretic. Madder is no longer used in medicine due to its cancerogenic potential and today it is better known as "Turkey Red" and for its role in the dye industry.

The root is aperient, astringent, cholagogue, diuretic and an emmenagogue. Taken internally, it is useful in the treatment of kidney and bladder stones. It is also reported to be effective in the treatment of amenorrhoea and jaundice. Its colour is sometimes used in osteopathic investigations as when taken internally, the root imparts a red colour to not only milk and urine, but the skeleton and in particular the developing bones of young individuals.

\section{Helichrysum}

Active ingredients: bitters, tannins, essential oil, flavonoids.

Uses: to stimulate digestive juices, strengthen the pancreas, anti-viral, anti-inflammatory and an analgesic. In homoeopathy to treat sciatica.

Warning: scarce - not to be collected.

Negative side effects: None known.

Helichrysum has been used medicinally as an expectorant in the treatment of colds. However, in homoeopathic medicine, a tincture proves very useful against sciatica, lumbago and some forms of arthritis. Steam distilled oil from this plant has a strong antiinflammatory action.

\section{Henbane}

Active ingredients: alkaloids hyoscyamine, scopolamine, atropine, tannins.

Uses: to treat asthma, colic and Parkinson's disease, to reduce muscular tension before surgery. Homeopaths use it to treat coughs and spasms and it was formerly used as a sedative and painkiller.

Warning: deadly poisonous.

Negative side effects: Due to the content of alkaloids, this is toxic if not taken in very low doses.

Henbane is a powerful brain relaxant, an antispasmodic on smooth muscle and a sedative. It inhibits the release of acetylcholine as a neurotransmitter, a similar action to that of Belladonna. Henbane is both an analgesic and a narcotic and has been used against Rabies, delirium associated with fevers, bronchitis, renal colic and coughs.

\section{Rough Bindweed}

Active ingredients: saponins, glycosides - sarsaponin, smilacin and pollinatanin (1.8-2.4\%), tannins, flavonoids, volatile oil, smilagenin.

Uses: occasionally with other medicines as a laxative, improves skin lesions and psoriasis.

Negative side effects: Like other saponin-containing plants this is poisonous taken in high doses. 
Recent research into the diseases referred to as Alzheimer's and Parkinson's - that is to say ageassociated memory impairment, have shown that one of the compounds found in rough bindweed, namely smilagenin, may be beneficial. Smilagenin, which is fat soluble and therefore better able to cross the bloodbrain barrier, has been shown to possess the ability to regulate brain receptors, in particular, to up-regulate M2 receptors in the brain, which are often found in low numbers in Alzheimer's and Parkinson's patients ${ }^{[5]}$. The saponins found in rough bindweed have been shown to facilitate the bodies absorption of other drugs and phytochemicals. The flavonoids in rough bindweed have been documented to have an immune modulation and liver protective effect, whilst, saponins have been shown to be very effective against psoriasis ${ }^{[6]}$.

Summary of ancient ailments: It seems likely, based on the medicinal properties of the plants understood in antiquity to pertain to Etruscan religious practice, that they would have been beneficial in the treatment of stomach and intestinal problems, skin lesions \& rashes, coughs \& colds, migraines \& headaches, arthritis and asthma.

Relevance to societal aspects: When looking back at the Etruscan society, it is worthwhile considering the diseases known to have been most prevalent at the time and therefore requiring treatment.

\section{Copper Smelting}

During the process of copper smelting, the most common industry, arsenic passes from the solid to the volatile state and leaves the smelting furnace in the form of localised toxic dusts ${ }^{[7]}$. The most common ailments associated with arsenic exposure would have been dyspepsia and respiratory inflammation. Another hazard may have been brought about by the handling of re-used water, used to cool the furnaces, giving rise to skin changes, lesions on the palms of the hand and soles of the feet. Other than these ailments, headaches, drowsiness, confusion and sometimes convulsions would have been common symptoms for those working with copper smelting.

Diet and Migraine: Food can play a significant role in the precipitation of headaches, although there is some evidence that there is a genetic prevalence towards migraine headaches, which can be inherited from generation to generation within a family. Cheese and red wine appear to be among the dietary substances that most frequently trigger an attack and cheese and wine were part of the daily diet.

Pollen and Asthma: Asthma and allergies have a strong hereditary and hence genetic component that likely works by modifying responses to ubiquitous environmental exposure. Indeed, increased exposure to both pollen and fungal spores is unequivocally associated with exacerbations of allergy and asthma. Both allergies and asthma were well-known in ancient society.

Diet, Stress and Arthritis: Non-genetic factors, such as aspects of lifestyle may explain approximately $40 \%$ of the cases of rheumatoid arthritis. In a recent study, it was found that a lower level of fruit intake and especially a lower level of vitamin $\mathrm{C}$, is associated with a greater than 3-fold increase in the risk of inflammatory arthritis ${ }^{[8]}$. Moreover, it has been found that a high dietary intake of red meat is associated with a greater risk of developing inflammatory arthritis, although it is unclear as to whether the association is a causative one ${ }^{[9]}$. Besides diet, lifestyle in relation to stress can play an important role in inflammatory arthritis. It has recently been shown that psychosocial factors, such as fearful experiences and parental disturbance during childhood can predispose to chronic musculoskeletal pain in later-life. These factors are probably just as relevant earlier in history as they are today.

\section{REFERENCES}

1. Jayaprakasam, B., N.P. Seeram and M.G. Nair, 2003. Anticancer and anti-inflammatory activities of cucurbitacins from Cucurbita andreana. Cancer Lett., 189: 11-16.

2. Roberfroid, M.B. and N.M. Delzenne, 1998. Dietary fructans. Annu. Rev. Nutr., 18: 117-143.

3. Saroglou, V., A. Karioti, C. Demetzos, K. Dimas and H. Skaltsa, 2005. Sesquiterpene Lactones from Centaurea spinosa and their antibacterial and cytotoxic activities. J. Nat. Prod., 68: 14041407.

4. Jain, N.K. and S.K. Kulkarni, 1999. Antinociceptive and anti-inflammatory effects of Tanacetum parthenium L. extract in mice and rats. J. Ethnopharmacol., 68: 251259.

5. Hu, Y., Z. Xia, Q. Sun, A. Orsi and D. Rees, 2005. A new approach to the pharmacological regulation of memory: Sarsasapogenin improves memory by elevating the low muscarinic acetylcholine receptor density in brains of memory-deficit rat models. Brain Res., 1060: 26-39.

6. Sampson, J.H., A. Raman, G. Karlsen, H. Navsaria and I.M. Leigh, 2001. In vitro keratinocyte antiproliferant effect of Centella asiatica extract and triterpenoid saponins. Phytomedicine, 8: 230235.

7. Arnott, R., 2005. Chrysokamino, Occupational Health and the Earliest Medicines in Crete. In Y. Tzedakis, H. Martlew and M. Jones (Eds.), Archaeology Meets Science: Biomolecular and Site Investigations in Bronze Age Greece, Oxford, Oxbow Books.

8. Kopec, J.A. and E.C. Sayre, 2004. Traumatic experiences in childhood and the risk of arthritis: A prospective cohort study. Can. J. Public Health, 95: 361-365.

9. Pattison, D.J., D.P.M. Symmons, M. Lunt, A. Welch, R. Luben, S.A. Bingham, K.T. Khaw, N.E. Day and A.J. Silman, 2004. Dietary risk factors for the development of inflammatory polyarthritis: Evidence for a role of high level of red meat consumption. Arthritis \& Rheumatism, 50: 38043812 . 